\title{
Assessing the Risk of a Canine Rabies Incursion in Northern Australia
}

\author{
Emily G. Hudson, Victoria J. Brookes and Michael P. Ward* \\ Sydney School of Veterinary Science, The University of Sydney, Camden, NSW Australia
}

OPEN ACCESS

Edited by:

Beatriz Martínez-López, University of California, Davis,

United States

Reviewed by:

Monique Sarah Léchenne, Swiss Tropical and Public Health

Institute, Switzerlana Louise Taylor,

Global Alliance for Rabies Control, United States

*Correspondence: Michael P. Ward michael.ward@sydney.edu.au

Specialty section: This article was submitted to Veterinary Epidemiology and Economics,

a section of the journal Frontiers in Veterinary Science

Received: 23 June 2017 Accepted: 14 August 2017 Published: 31 August 2017

Citation:

Hudson EG, Brookes VJ and Ward MP (2017) Assessing the Risk of a Canine Rabies Incursion in Northern Australia.

Front. Vet. Sci. 4:141. doi: 10.3389/fvets.2017.00141
Rabies is a globally distributed virus that causes approximately 60,00 human deaths annually with $>99 \%$ of cases caused by dog bites. Australia is currently canine rabies free. However, the recent eastward spread of rabies in the Indonesian archipelago has increased the probability of rabies entry into northern Australian communities. In addition, many northern Australian communities have large populations of free-roaming dogs, capable of maintaining rabies should an incursion occur. A risk assessment of rabies entry and transmission into these communities is needed to target control and surveillance measures. Illegal transportation of rabies-infected dogs via boat landings is a high-risk entry pathway and was the focus of the current study. A quantitative, stochastic, risk assessment model was developed to evaluate the risk of rabies entry into north-west Cape York Peninsula, Australia, and rabies introduction to resident dogs in one of the communities via transport of rabies-infected dogs on illegal Indonesian fishing boats. Parameter distributions were derived from expert opinion, literature, and analysis of field studies. The estimated median probability of rabies entry into northwest Cape York Peninsula and into Seisia from individual fishing boats was $1.9 \times 10^{-4}$ / boat and $8.7 \times 10^{-6} /$ boat, respectively. The estimated annual probability that at least one rabies-infected dog enters north-west Cape York Peninsula and into Seisia was $5.5 \times 10^{-3}$ and $3.5 \times 10^{-4}$, respectively. The estimated median probability of rabies introduction into Seisia was $4.7 \times 10^{-8} /$ boat, and the estimated annual probability that at least one rabies-infected dog causes rabies transmission in a resident Seisia dog was $8.3 \times 10^{-5}$. Sensitivity analysis using the Sobol method highlighted some parameters as influential, including but not limited to the prevalence of rabies in Indonesia, the probability of a dog on board an Indonesian fishing boat, and the probability of a Seisia dog being on the beach. Overall, the probabilities of rabies entry into north-west Cape York Peninsula and rabies introduction into Seisia are low. However, the potential devastating consequences of a rabies incursion in this region make this a non-negligible risk.

\section{Keywords: risk, rabies, Australia, dogs, surveillance}

\section{INTRODUCTION}

Rabies is a vaccine-preventable viral infection that is globally distributed and causes approximately 59,000 human deaths annually (1). Although all mammals can be infected by rabies, domestic dogs are responsible for $>99 \%$ of human cases (2). In recent years, rabies has spread into rabies-free regions of the world such as areas in Indonesia $(3,4)$, Bhutan $(5,6)$, and China $(7,8)$. Spread has predominately been attributed to transportation of rabies-infected dogs. Effective surveillance is critical for timely 
detection of rabies incursions. Detection time can subsequently affect the outcome of a rabies incursion from elimination if detected early to endemic infection following delayed detection (9). Surveillance and biosecurity measures (for example, quarantine, import restrictions, or coastal patrols) are also important in rabiesfree regions to prevent potential rabies incursions. However, with limited resources, surveillance efforts and control measures must be targeted to areas that have the greatest effect on limiting rabies entry and transmission (10); this is also likely to be cost-efficient.

Risk assessment and subsequent sensitivity analysis are useful tools to assess the likelihood of disease incursions and direct surveillance efforts and control measures (10). There are several recent examples of such studies, in which the risk of entry of rabies via legal routes that are controlled by import restrictions have been assessed. For example, a rabies entry risk assessment for the United Kingdom found that a change in importation policy from the current UK Pet Travel Scheme (PETS) to the European Pet Movement Policy would result in a 60-fold increase in rabies entry risk. Sensitivity analysis demonstrated that non-compliance with control measures was a highly influential parameter (11). Noncompliance with control measures-such as improper vaccination or serological protocol and document forgery-has also been found to be influential in earlier assessments of the risk of rabies entry associated with the PETS scheme $(12,13)$. Another risk assessment of rabies entry into Taiwan also highlighted non-compliance as an influential parameter (14). It has been suggested that illegal smuggling is likely to pose a higher risk of rabies incursion compared to legal modes of transport; in some cases, if illegal smuggling is not reduced to almost zero, the total risk of rabies entry cannot be lowered to an acceptable level (14). Illegal transportation of infected dogs via boat has already been responsible for the spread of rabies into previously rabies-free regions of Indonesia such as Flores and Bali $(4,15,16)$ and is considered to be a high-risk entry pathway of rabies into other rabies-free areas in the region $(17,18)$.

Australia is currently canine rabies free. However, the eastward spread of rabies through the Indonesian archipelago has reduced the distance between northern Australian regions, such as the Northern Peninsula Area (NPA) and rabies-infected islands in Indonesia (3). There are cultural connections between Indonesia, Papua New Guinea (PNG), the Torres Strait, and northern mainland Australia, which subsequently cause human movements between the four areas. These movements increase the probability of a rabies incursion into northern Australian communities such as the NPA. In addition, the coastline of northern Australia is vast-approximately $10,000 \mathrm{~km}$ between Broome in the west and Cairns in the east (www.agriculture.gov.au/biosecurity/ australia/naqs) - and is sparsely populated. Surveillance of the entire region is difficult, which provides opportunity for unregulated boat landings. Deriving parameters for risk assessment of illegal routes of entry is challenging due to the covert nature of illegal activities and subsequent lack of published information. Structured methods to elicit parameters from experts with local knowledge is one source that can be used to provide information for inputs with unknown values $(19,20)$. In areas in which there are communities such as the NPA, there is also opportunity for rabies establishment following entry due to the large population of free-roaming domestic dogs that reside within such communities
(21). Risk assessments that include exposure and transmission of rabies to resident dogs can highlight postborder control areas to minimize the risk of rabies establishment, following an incursion. For example, the probability of dog confinement following entry of an infected dog was an influential parameter in a risk assessment model for rabies introduction into Lombok, Indonesia, and it was suggested that it could be targeted to minimize rabies transmission risk (22). Similarly, a risk assessment for rabies establishment risk in the port of Hokkaido, Japan, from illegal landings of Russian fishing boats found that the probability of contact with wildlife was a highly influential factor and could be targeted to minimize the predicted risk (23).

The risk of rabies entry and transmission to resident dogs in the NPA has not been previously estimated. In this study, we identified potential illegal routes of entry using qualitative methods and then used a quantitative risk assessment model to evaluate the risk of rabies entry into the NPA and rabies entry and transmission to resident dogs in a community in the NPA. The model used parameter estimates derived during a workshop using structured methods to elicit expert opinion, as well as information from field data and literature. A sensitivity analysis was conducted to identify factors that influenced rabies risk. The findings from this study will be used to direct surveillance and control measures to mitigate the risk of rabies introduction in this region.

\section{MATERIALS AND METHODS}

\section{Definition of Risk Pathways}

Structured interviews to define possible risk pathways for entry of a rabies-infected dog into Cape York Peninsula (direct from Indonesia or via PNG and the Torres Strait) were conducted in August 2016 prior to an expert opinion workshop. Biosecurity officers and Land and Sea Rangers $(n=8)$ were purposefully selected as key informants due to their local knowledge and collectively extensive experience of biosecurity-associated activities in the Cape York Peninsula region. Risk pathways considered in the interviews included the mode of transport (boat or plane), direct or indirect travel (from Indonesia or through PNG and the Torres Strait), reason for travel, and landing sites in the Cape York Peninsula. The highest risk pathways for further investigation using quantitative methods were selected based on agreement between key informants.

Key informants confirmed that illegal boats were the likely mode of transport of dogs from Indonesia to Cape York Peninsula. The main reason for travel of Indonesian people was believed to be fishing, specifically for shark-fin. The main reasons for a dog on board a fishing boat was for hunting and companionship. They also stated that these boats travel directly from Indonesia to Northern Australia and arrive along the Cape York Peninsula coast and do not travel via PNG and the Torres Strait. Informants described that likely locations of entry were in remote areas along the north-western coastline of Cape York Peninsula between the northern section of the NPA $\left(10.6693^{\circ} 142.533^{\circ} \mathrm{E}\right)$ and south to the mouth of the Skardon River $\left(11.757^{\circ} \mathrm{S} 142.002^{\circ} \mathrm{E}\right.$; a distance of approximately $122 \mathrm{~km}$; Figure 1). This area is subsequently referred to as north-west Cape York Peninsula. Informants stated that the likely entry point of Indonesian boats specifically into the NPA communities was at Seisia. 
Two scenarios were selected for quantitative risk assessment: Scenario 1 - the entry of a rabies-infected dog to north-west Cape York Peninsula (including the NPA) via an illegal fishing boat from Indonesia, which stays for a few days; Scenario 2-exposure and infection of a resident, domestic dog following entry of a rabies-infected dog to the beach community of Seisia.

We considered the following pathway for Scenario 1. An illegal fishing boat leaves Indonesia and is carrying at least one rabiesinfected dog. The infected dog (or dogs) survives the journey to Australia and disembarks the boat. Exposure and transmission to a resident dog is not included in this scenario. Scenario 2 refines and extends Scenario 1; an illegal Indonesian fishing boat traveling to north-west Cape York Peninsula lands briefly on the beach at Seisia (Figure 1). An Indonesian, rabies-infected dog disembarks the boat and comes into effective contact with at least one resident, domestic dog that subsequently develops rabies. The boat and the Indonesian dog leave Seisia.

By using the generic scenario tree developed by Ward and Hernández-Jover (10) as a framework, a scenario tree was developed to describe the pathways (Figures 2 and 3). Nodes

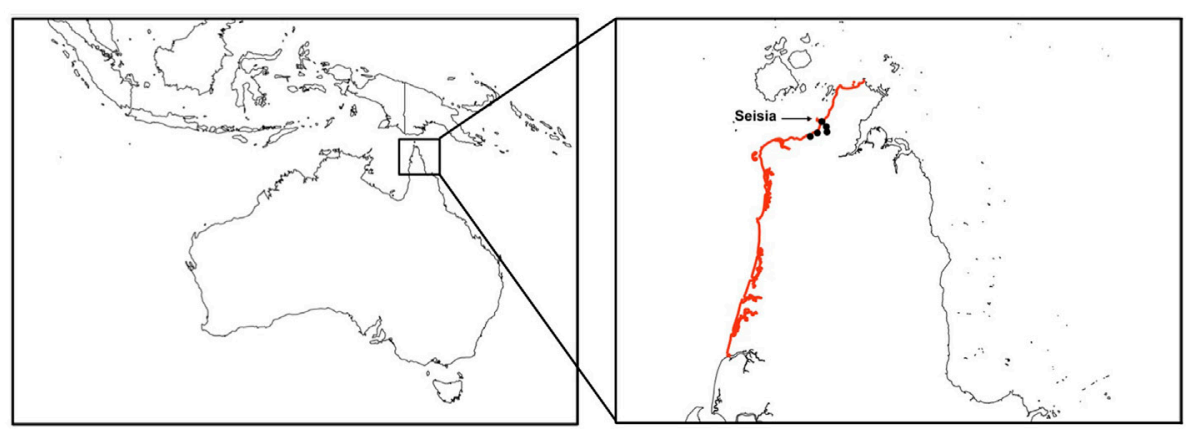

FIGURE 1 | Cape York Peninsula, Australia, with the region of entry of Indonesian fishing boats considered in a risk assessment of the entry of a rabies-infected dog highlighted. This region includes the five communities of the Northern Peninsula Area. The community of Seisia is labeled.

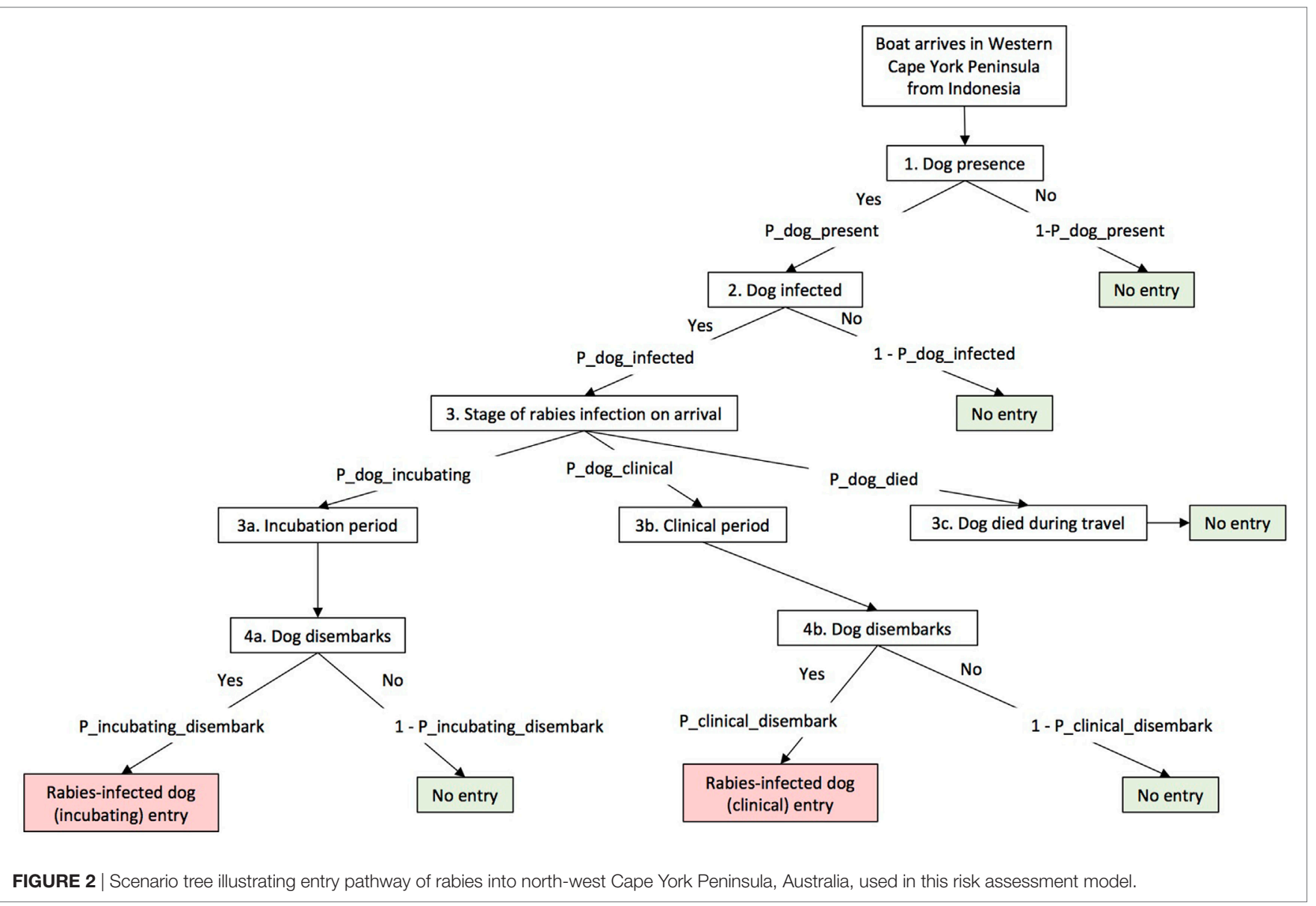




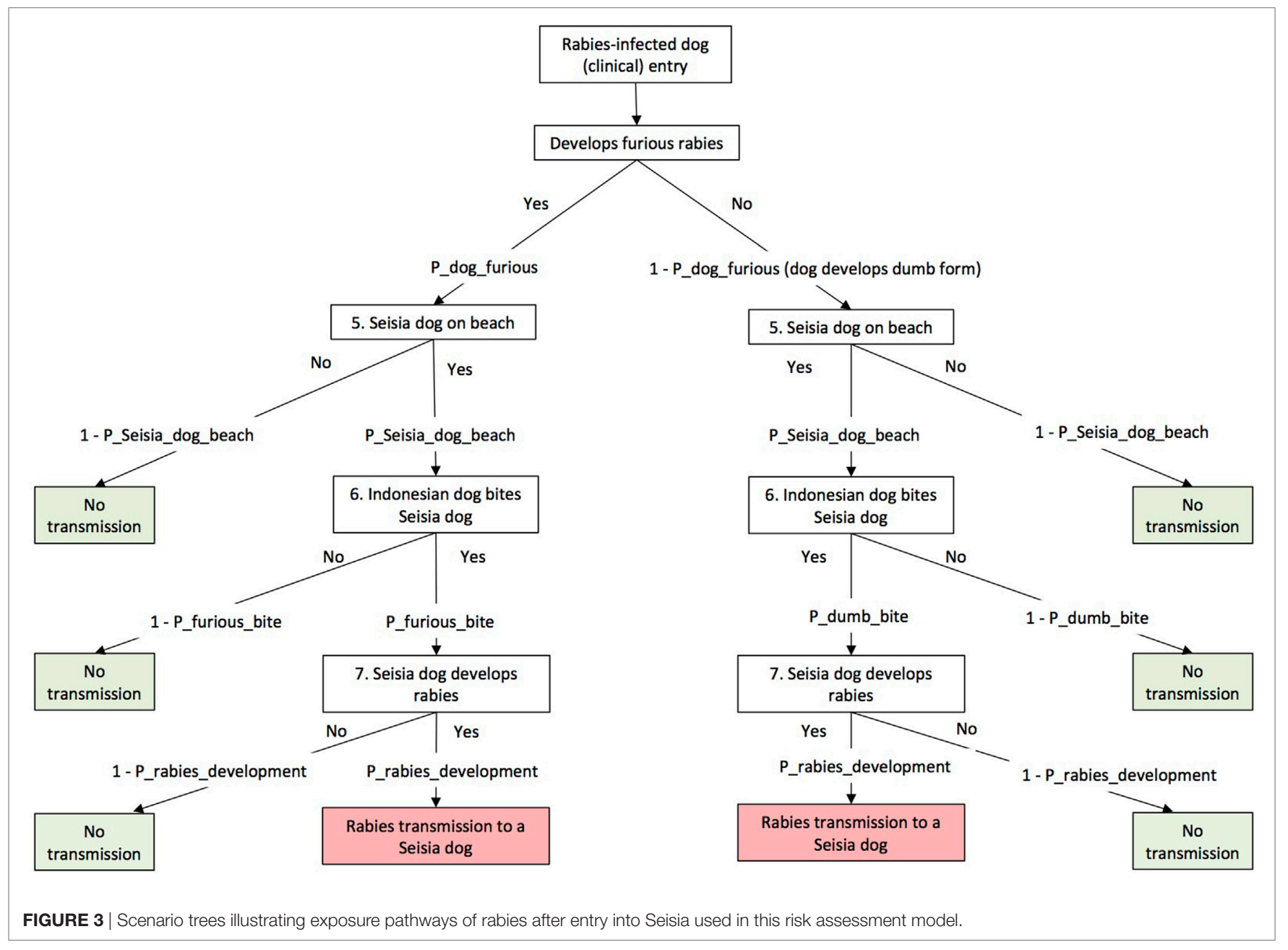

1-4 relate to the entry assessment into north-west Cape York Peninsula (including Seisia), and Nodes 5-7 relate to the assessment of rabies exposure and subsequent transmission a dog in Seisia (Scenario 2). The nodes were described using 22 parameters of which 8 were derived from the literature, 12 from an expert opinion workshop conducted in August 2016, and 2 from the analysis of field data. Assumptions, nodes, and parameters are described in detail below and in Table 1. Quantitative, stochastic, risk assessment models were developed to evaluate these pathways. Model outputs are described below. The sensitivity of these outputs to model inputs was assessed using the Sobol method (also described below).

\section{Model Outputs}

The following model outputs were derived using Monte Carlo simulation (10,000 iterations) of quantitative, stochastic models constructed for each scenario; probability of rabies entry into north-west Cape York Peninsula (Scenario 1) and entry into Seisia (Scenario 2) via an individual Indonesian fishing boat; annual number of rabies-infected dogs and the probability of entry of at least one rabies-infected dog entering per year in north-west Cape York Peninsula (Scenario 1) and Seisia (Scenario 2); the probability of rabies entry and transmission (introduction) into Seisia (Scenario 2) via an individual Indonesian fishing boat; and finally, the probability of introduction from at least one rabiesinfected dog per year in Seisia (Scenario 2). Also calculated is the time until next event for each scenario-the number of years for one rabies-infected dog to enter north-west Cape York Peninsula (Scenario 1) and Seisia (Scenario 2) as well as the number of years for one rabies-infected to dog to transmit rabies to a resident Seisia $\operatorname{dog}$ (Scenario 2). This is estimated by a gamma distribution using the respective number of infected dogs per year for each scenario.

\section{Expert Opinion Workshop Overview}

Following structured interviews and description of entrypathways, an expert opinion workshop was conducted to derive parameters to assess the risk of entry of rabies-infected dogs to north-west Cape York Peninsula and Seisia. Workshop participants were 13 NPA community members -7 Land and Sea Rangers, 3 Biosecurity Officers, a manager from the Northern Peninsular Area Regional Council, an Environmental Management Worker, and the manager of the local community enterprise business. The distribution of ages and expertise of participants are shown in 
TABLE 1 | List of model parameters and their values for a risk assessment of canine rabies entry via Indonesian fishing boats into north-west Cape York Peninsula and rabies introduction to a resident dog in Seisia, Queensland, Australia.

\begin{tabular}{|c|c|c|c|c|}
\hline Node & Parameter & Description & Value & Source \\
\hline 1 & Probability boat carrying at least one dog & PERT (min, most likely, max) & $0.0217,0.1196,0.4329$ & Expert opinion \\
\hline 2 & Prevalence of rabies in Indonesia $(0-1)$ & Uniform (min, max) & $0.0001,0.05$ & $\begin{array}{l}\text { Kitala et al. (24); Cleaveland et al. (25); } \\
\text { Kayali et al. (26); Tenzin et al. (5); Tenzin } \\
\text { et al. (6); Mustiana (22); Putra et al. (4) }\end{array}$ \\
\hline \multirow[t]{6}{*}{3} & Incubation period (days) & Lognormal (mean, SD) & $23.6,17.3$ & Tojinbara et al. (27) \\
\hline & Clinical period (days) & Gamma (shape, rate) & $2.83,0.91$ & Hampson et al. (28) \\
\hline & Duration of travel (days) & Empirical (min, median, max) & $1.092,4.711,24.61$ & Expert opinion \\
\hline & Probability arrives in incubation period & Empirical (min, median, max) & $0.6386,0.6528,0.6715$ & Derived from expert opinion parameters \\
\hline & Probability arrives in clinical period & Empirical (min, median, max) & $0.1548,0.1708,0.1809$ & Derived from expert opinion parameters \\
\hline & Probability dies during travel & Empirical (min, median, max) & $0.1654,0.1764,0.1902$ & Derived from expert opinion parameters \\
\hline \multirow[t]{5}{*}{4} & Duration of stay (days) & Empirical (min, median, max) & $0.956,5.177,109.7$ & Expert opinion \\
\hline & $\begin{array}{l}\text { Probability apparently healthy dog disembarks } \\
<5 \text { days stay }\end{array}$ & PERT (min, most likely, max) & $0.0213,0.0851,0.1$ & Expert opinion \\
\hline & $\begin{array}{l}\text { Probability apparently healthy dogs disembarks } \\
\geq 5 \text { days stay }\end{array}$ & PERT (min, most likely, max) & $0.0213,0.0851,0.319$ & Expert opinion \\
\hline & Combined probability apparently healthy dog disembarks & Empirical (min, median, max) & $0.023,0.08545,0.300$ & Derived from expert opinion parameters \\
\hline & Probability a clinically affected dog disembarks & PERT (min, most likely, max) & $0.021,0.021,0.051$ & Expert opinion \\
\hline \multirow[t]{3}{*}{5} & Probability a boat lands on Seisia beach & Point value & 0.0455 & Expert opinion \\
\hline & Total daily beach-minutes (minutes) & Empirical (min, median, max) & $141,379,7,710$ & Field data \\
\hline & Daily probability that a resident dog is on Seisia beach & Empirical (min, median, max) & $0.089,0.264,0.556$ & Field data \\
\hline \multirow[t]{4}{*}{6} & Probability dog develops furious rabies & Uniform (min, max) & $0.1,0.6$ & $\begin{array}{l}\text { Vaughn et al. (29); Fekadu and } \\
\text { Shaddock (30); Foggin (31) }\end{array}$ \\
\hline & Probability dog develops dumb rabies & Uniform (min, max) & $1-$ Uniform $(0.1,0.6)$ & $\mathrm{n} / \mathrm{a}$ \\
\hline & Probability of dog bite if dumb form & Uniform (min, max) & $0.001,0.1$ & Field data \\
\hline & Probability of dog bite if furious form & Uniform (min, max) & $0.5,0.8$ & Hampson et al. (28) \\
\hline 7 & Probability of rabies development after bite & Uniform (min, max) & $0.35,0.52$ & Hampson et al. (28) \\
\hline \multirow[t]{3}{*}{8} & $\begin{array}{l}\text { Number of boats that arrive the north-west Cape York } \\
\text { Peninsula/year }\end{array}$ & Empirical (min, median, max) & $1,6,23$ & Expert opinion \\
\hline & Number of boats that arrive in the NPA (Seisia)/year & Empirical (min, median, max) & $\begin{array}{l}0.0455 \times \text { empirical } \\
(1,6,23)\end{array}$ & Derived from expert opinion parameters \\
\hline & Number of dogs per boat & Empirical (min, median, max) & $1,2,5$ & Expert opinion \\
\hline
\end{tabular}

Probabilities are given in the range $0-1$.

Table 2. A modified Delphi technique and participatory methods (proportional piling) were used to elicit information from the participants.

\section{Delphi Process}

A four-step elicitation procedure was used to elicit values and limit overconfidence of estimates (20). Participants were asked to individually estimate the minimum, most likely, and maximum values as well their confidence that their minimum-maximum interval contained the true value. This procedure was used to gather information about the number of boats arriving at northwest Cape York Peninsula, the duration of travel from Indonesia, duration of stay in north-west Cape York Peninsula, and the numbers of dogs per boat. The experts' intervals were standardized to $80 \%$ confidence intervals using Eqs 1 and 2, in which $\alpha_{a b s}$ is the standardized minimum estimate, $\alpha$ is the expert's minimum estimate, $\gamma$ is the expert's most likely estimate, $\beta_{\mathrm{abs}}$ is the standardized maximum estimate, $\beta$ is the expert's maximum estimate, $c$ is the expert's confidence level, and $p$ is the standard confidence interval $(19,32)$. The distributions of expert opinions were combined to create empirical distributions for each parameter.

$$
\alpha_{\mathrm{abs}}=\gamma-(\gamma-\alpha)(c / p)
$$

TABLE 2 | Expert opinion participant information from a workshop conducted in 2016 to parameterize a risk assessment of rabies entry via Indonesian fishing boats into north-west Cape York Peninsula and rabies introduction to a resident dog in Seisia, Queensland, Australia.

\begin{tabular}{lclc}
\hline Participant occupation & Age & Sex & Years in current job \\
\hline Biosecurity officer & $20-30$ & Male & $<1$ \\
Biosecurity officer & $30-40$ & Male & $1-7$ \\
Biosecurity officer & $40-50$ & Male & $8-15$ \\
Council manager & $50-60$ & Female & $1-7$ \\
Council manager & $50-60$ & Male & $8-15$ \\
Land and sea ranger & $20-30$ & Female & $<1$ \\
Land and sea ranger & $18-20$ & Female & $<1$ \\
Land and sea ranger & $20-30$ & Male & $<1$ \\
Land and sea ranger & $30-40$ & Male & $1-7$ \\
Land and sea ranger & $20-30$ & Male & $1-7$ \\
Land and sea ranger & $20-30$ & Male & $1-7$ \\
Land and sea ranger & $30-40$ & Male & $1-7$ \\
Local business manager & $50-60$ & Male & $1-7$ \\
\hline
\end{tabular}

$$
\beta_{\text {abs }}=\gamma+(\beta-\gamma)(c / p)
$$

\section{Proportional Piling}

Proportional piling was used in group exercises in which participants openly discussed probabilities and counts associated 
with the scenarios and illustrated their opinions using piles of jellybeans (33). Participants used this method to demonstrate the numbers of boats that land at locations in Cape York Peninsula. They also used proportional piling to describe the minimum, most likely, and maximum values for the proportion of boats that carry dogs and the proportion of dogs that disembark (dependent on disease progression and whether dogs appeared sick or healthy). These values were used to create PERT distributions for each parameter.

\section{Sensitivity Analysis}

Sensitivity analyses were performed using variance-based global sensitivity analysis and the Sobol method from the "sensitivity" package in $\mathrm{R}$ (34). We assessed one outcome for each scenariothe annual number of infected dogs from Indonesia that enter north-west Cape York Peninsula (Scenario 1) and the probability that a rabies-infected dog arrives via boat from Indonesia and infects a resident Seisia dog (Scenario 2) following the landing of an Indonesian boat in Seisia. The Sobol method estimates each input distribution's influence on the variance of the outcome variable both individually ("main" or "first-order" effect) and with interactions with other inputs ("total" effect). Sensitivity indices (SIs) were estimated for each input's main and total effects; the higher the SI, the more influence the distribution has on the outcome. Plots of total and main effect SIs for each scenario outcome were created with $95 \%$ confidence intervals estimated using the "boot" package in $\mathrm{R}(35,36)$.

\section{Parameter Information Annual Number of Boats Entering North-West Cape York Peninsula (Scenario 1) and Seisia (Scenario 2)}

An empirical distribution of the number of boats arriving in north-west Cape York Peninsula was estimated using the modified Delphi technique to elicit values in the expert opinion workshop. A binomial distribution with the probability of a Seisia landing (determined using proportional piling) and the annual number of boats entering north-west Cape York Peninsula was used to describe the annual number of boats entering Seisia.

\section{Number of Dogs Entering per Boat in North-West Cape York Peninsula (Scenario 1) and Seisia (Scenario 2)}

The modified Delphi technique during the expert opinion workshop was used to derive the number of dogs per boat with at least one dog. Seisia is part of north-west Cape York Peninsula; therefore, the same distribution was used for boats entering Seisia (Scenario 2).

\section{Node 1-Probability of a Dog on Board a Fishing Boat from Indonesia}

The probability of at least one dog on board an Indonesian fishing boat that arrives in north-west Cape York Peninsula was determined in the expert opinion workshop using proportional piling. The minimum, most likely, and maximum values given were used to create a PERT distribution. This probability was also used for boats landing in Seisia community (Scenario 2).

\section{Node 2-Probability That a Dog Is Infected with Rabies}

The probability that a dog is infected with rabies depends on the prevalence of rabies at its origin in Indonesia. Rabies prevalence or incidence varies throughout endemic regions in Indonesia and is difficult to measure due to the potentially long incubation period of rabies and surveillance constraints. For example, the monthly attack rate of rabies in dogs (laboratory confirmed cases) in an outbreak in Bali was estimated to be 0.0003 prior to vaccination campaigns (4). Another study estimated a prevalence of 0.005 in Flores and 0.01 in South Sulawesi (22). Similarly low prevalence or incidence rates have been reported in other rabies endemic regions such as in Tanzania, Kenya, and Chad, where reported incidence of rabies in unvaccinated dogs has ranged between 0.0014 and 0.01 (24-26). Even in outbreak situations-such as that seen in Bhutan in the eastern districts in 2005-2007 and in the Chukha District in 2008-incidence can still be low $(0.0143$ and 0.02 , respectively) $(5,6)$. Considering these low but varying estimates in regions around the world, a uniform distribution of 0.0001-0.05 was used in this risk assessment to describe the probability that a dog was infected with rabies.

\section{Node 3-The Probability of Surviving Travel}

The probability that the dog survives the journey and its stage of disease is dependent on the duration of travel from Indonesia and the length of incubation and clinical periods. The duration of travel was described by an empirical distribution derived from the expert opinion workshop. The minimum duration of travel was assumed to be no less than 1 day; therefore, the derived empirical distribution was truncated to reflect this. The incubation period depends on virus strain, the anatomical location of the bite, and virus dose. A study of 98 rabies cases in dogs from data collected in Tokyo, Japan, estimated a mean incubation period of 27 days (SD 20 days) (27). By using these data, we reproduced a lognormal distribution to describe the incubation period in this risk assessment (median 19 days; 95\% percentile 5.4-68.6 days). A gamma distribution was used to describe the duration of the clinical period based on reconstructed case histories of over 1,000 suspected rabid dogs in Tanzania (28). We reproduced this distribution to describe the duration of clinical period in the risk assessment model (mean 3.1 days; 95\% percentile 0.6-7.7 days).

The probability that a dog survived travel and its stage of infection on arrival was based on the following simulation. An incubation period, duration of travel, and duration of clinical signs were randomly selected from their respective distributions. The duration of disease was the combined incubation and clinical periods. We assumed that a dog showing clinical signs would not be taken aboard a boat in Indonesia. Therefore, the day of disease progression on which the dog started its journey to Australia was randomly chosen from the incubation period. The day of disease progression on arrival in Australia was calculated from the travel duration plus the randomly chosen start day. If the day of disease progression on arrival was greater than the duration of infection, the dog died during travel. If the day of disease progression on arrival was less than the incubation period, the dog arrived during the incubation period. If the day of disease progression on arrival was less than the duration of disease but greater than the 
incubation period, the dog arrived within the clinical period. The number of dogs in each category were counted, and a distribution for the probability of a dog arriving in each category was derived.

\section{Node 4-Probability That a Rabies-Infected Dog Disembarks in Australia}

This node describes the probability that a rabies-infected dog disembarks from the boat once it has arrived in north-west Cape York Peninsula. Workshop participants stated that the probability of disembarkation will be dependent on whether the dog is in the clinical phase or still incubating and not showing clinical signs. The duration of stay of the fishermen once arrived in north-west Cape York Peninsula could also affect this probability. For example, higher probabilities of disembarkation might be associated with longer duration of stay. The modified Delphi technique was used to derive the duration of stay in north-west Cape York Peninsula. Proportional piling during the workshop was used to estimate the probability of dog disembarkation if the dog was either showing or not showing clinical signs, for durations of stay of $<5$ days and $\geq 5$ days (the 5 -day threshold was defined by the participants of the workshop). The two probabilities for each disease stage were then combined by randomly selecting a value from the duration of stay distribution and then randomly selecting a value for the probability of disembarkation from the relevant probability distribution (either $<5$ or $\geq 5$ days).

\section{Node 5-Probability That a Rabies-Infected Indonesian Dog Meets a Resident Dog}

The probability that a rabies-infected dog that arrives on an illegal fishing boat from Indonesia meets a resident NPA dog is dependent on the time that resident dogs are present on Seisia beach. We used the term "beach-minutes" to describe the number of minutes of each day that a resident dog could be expected to be on the Seisia beach. The total beach-minutes per day was estimated from previously collected GPS data of 29 dogs in Seisia [September 2013 ( $n=9$ dogs), April and September $2014(n=8$ and 7 dogs, respectively) and June 2015 ( $n=5$ dogs)] for which the GPS fix interval was set at $1 \mathrm{~min}$ (37). From these datasets, the number of beach-minutes for each dog was estimated using a GPS coordinate error of $18 \mathrm{~m}$. The median time-gap between GPS locations was added to each beach-trip to adjust for the time spent on the beach before and after the recorded GPS fixes. Poisson distributions were used to describe variability of each dog's daily beach-minutes.

Of the 29 dogs collared, 20 dogs (68\%) visited the beach at least once; therefore, we made the assumption that approximately two-thirds of the current population would spend time on the beach. The current population of dogs in Seisia is approximately 50 dogs (21). Therefore, 30 Poisson distributions were randomly selected (with replacement) and combined to simulate the daily beach-minutes of the dogs that visit the beach in Seisia. This was repeated 100 times, and the resulting distributions were combined to produce an overall distribution of daily beach-minutes. The probability that a resident dog would be on the beach when a rabies-infected dog arrives on an Indonesian fishing boat was described by a beta distribution derived from the overall beachminutes distribution.

\section{Node 6-Probability an Indonesian Dog Bites a Resident Dog}

The probability that a clinically affected rabies-infected Indonesian dog bites a resident Seisia dog is likely to differ depending on whether the dog has developed the dumb or furious form of rabies. We assumed that the probability a dog with the dumb form of rabies bites another dog is likely to be similar to background dog bite incidence. By using data collected from a survey of 178 dogs in May 2016, a background probability of receiving a bite between NPA dogs was estimated as 0.00693 (38 dogs received a bite during the previous month). This probability is likely to be an underestimate due to recall bias (the owners surveyed did not remember all the dog bites) or lack of observation of minor bites. Also, these bites are between NPA dogs and do not account for the possibility that bite probability could be increased due to aggression between unfamiliar dogs. This estimate is also the probability of a dog receiving a bite - not the probability of a dog biting another dog-and so to account for these uncertainties a uniform distribution of $0.001-0.1$ was used to describe the daily bite probability of a dog with the dumb form of rabies. If the dog has developed furious rabies, the probability that it bites another dog is likely to be higher. The probability of developing furious rabies is variable. Observational studies in which samples of suspect animals are sent for testing have suggested that high proportions (up to 0.75 ) of rabies-infected dogs develop furious rabies (31). However, these studies are likely to overestimate this proportion due to reporting bias (furious cases are more likely to be recognized and submitted for testing). Experimental testing has demonstrated a lower proportion (between 0.05 and 0.4 ) of furious rabies development $(29,30,38)$. In the current study, the probability of developing furious rabies was therefore assumed to be a uniform distribution $0.1-0.6$. The bite probability of a dog with the furious form is likely to be high due to the aggressive behavior associated with this form of rabies. Hampson et al. (28) estimated dog bites using a negative binomial distribution of mean 2.15 bites/rabid dog (95\% CI 1.95-2.37) and clinical period 3.1 days (95\% CI 2.9-3.4). This produces an approximate mean daily bite probability of 0.7 . We assumed that most dogs in the study by Hampson et al. (28) developed the furious form because dogs suffering from the furious form would be more likely to be observed and therefore used to calculate bite probability. Therefore, we used a uniform distribution $0.5-0.8$ around the approximate mean calculated from the study by Hampson et al. (28) to describe the probability of a bite if the dog developed furious rabies.

\section{Node 7-Probability That a Resident Dog Develops Rabies following a Bite}

Australia is canine rabies free; therefore, dogs are unvaccinated. There is natural variability in the probability of rabies development after a dog bite, dependent on virus strain, the anatomical location of the bite, and virus dose. Hampson et al. (28) estimated that the probability that an unvaccinated dog would develop rabies following a bite from a rabies-infected animal was 0.49 (95\% CI $0.45-$ 0.52 ). It is possible that severe bites were more likely observed. The probability of rabies transmission might be lower following a less severe bite in which less virus is transferred. Therefore, a uniform 
distribution of $0.35-0.52$ was used to describe the probability of rabies infection following a bite from a rabid animal.

\section{RESULTS}

\section{Parameter Information}

All distributions used in the model are described in Table 1.

\section{Number of Boats Arriving in the North-West Cape York} Peninsula (Scenario 1) and Seisia (Scenario 2) per Year The median number of Indonesian fishing boats (regardless of whether a dog is on board) arriving in north-west Cape York Peninsula per year was estimated to be 6 (95\% percentile 3-17 boats) based on the combined individual PERT distributions from workshop information (Figure 4A). Workshop participants estimated that the proportion of the boats that traveled to northwest Cape York Peninsula and landed in Seisia was 0.0455. Therefore, the median number of Indonesian fishing boats landing in Seisia was 0 boats per year (95\% percentile $0-2$ boats).

\section{Number of Dogs per Boat Arriving in North-West Cape York Peninsula (Scenario 1) and Seisia (Scenario 2)}

The median number of dogs on boats with at least one dog was 2 dogs per boat (95\% percentile 1-5 dogs/boat; Figure 4B).

\section{Node 1-Probability of at Least One Dog on Board a Fishing Boat from Indonesia}

Estimates generated by the workshop participants $(0.02,0.12$, and 0.43 for minimum, most likely, and maximum probabilities, respectively, of at least one dog on board a fishing boat from
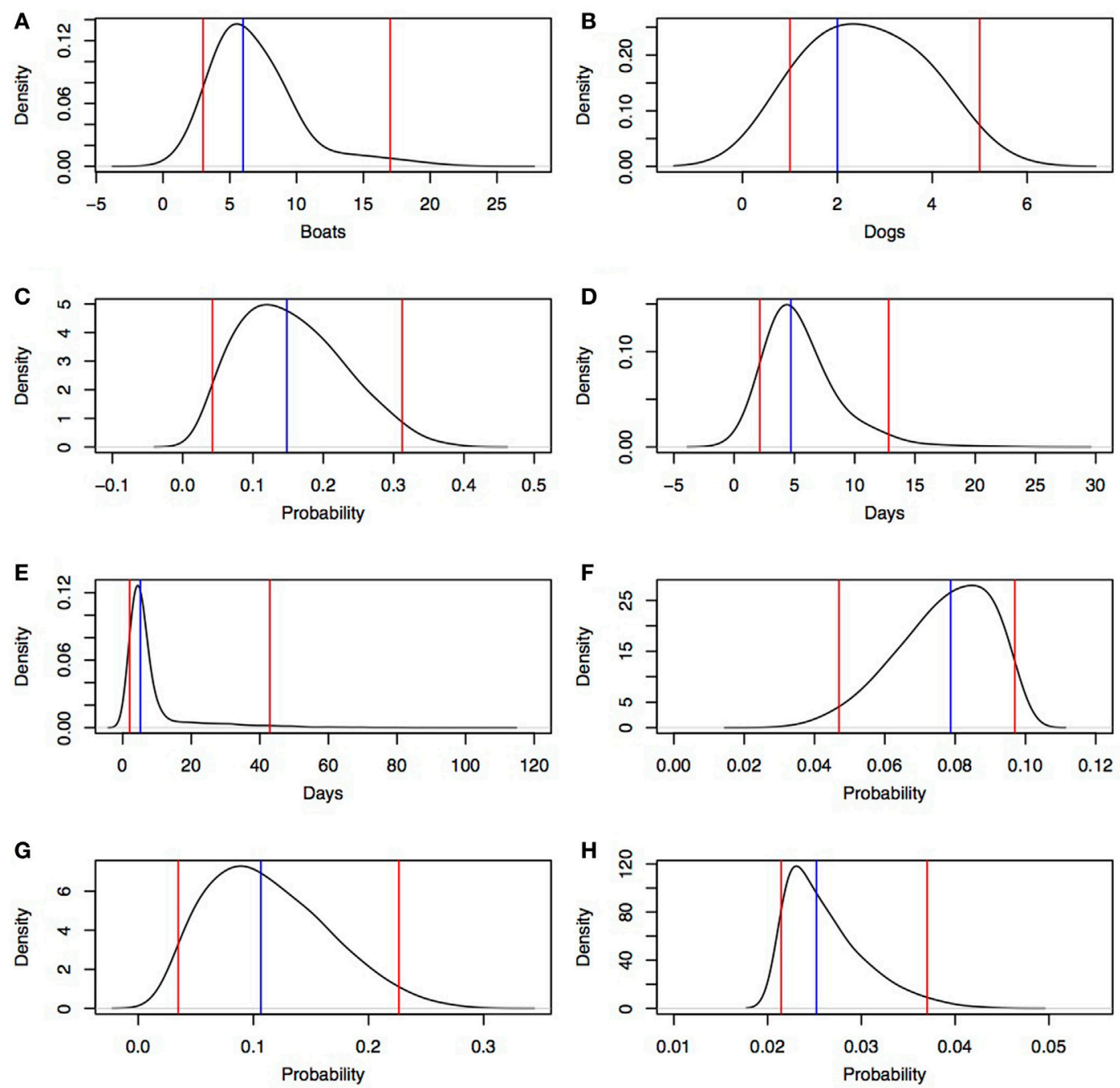

FIGURE 4 | Density distributions derived from an expert opinion workshop in a rabies risk assessment, north-west Cape York Peninsula, Australia. Blue lines, median; red lines, 95\% percentile. (A) number of boats arriving at north-west Cape York Peninsula per year, (B) number of dogs per boat for boats with at least one dog, (C) probability an Indonesian fishing boat has at least one dog, (D) duration of travel from Indonesia to north-west Cape York Peninsula, (E) duration of stay of Indonesian fishermen after landing, (F) probability an apparently healthy dog disembarks if staying $<5$ days, (G) probability an apparently healthy dog disembarks if staying $\geq 5$ days, and $\mathbf{( H )}$ probability a clinically affected dog disembarks. 
Indonesia) were used to create a PERT distribution (Figure 4C). Participants stated that variability in these probabilities depended on whether the fishermen owned a dog and the secondary activities of the fisherman once they arrived in Australia (for example, if hunting was expected, they were more likely to bring dogs).

\section{Node 3-Probability of Stage of Rabies Infection on Arrival}

The median duration of travel from Indonesia to north-west Cape York Peninsula was 4.7 days (95\% percentile 2.1-12.8 days; Figure 4D). Workshop participants noted that duration of travel is dependent on origin in Indonesia and weather conditions. The simulation produced three probabilities; two probabilities for a dog that has survived the journey to north-west Cape York Peninsula [one for each arrival disease state-incubating (not showing clinical signs)] and clinical (showing clinical signs) and one for the probability the dog died from rabies during travel. The most likely outcome was that a dog arrives during its incubation period (median 0.65 ; 95\% percentile $0.64-0.66$ ); lower probabilities were estimated for a dog dying of rabies during travel (median 0.18 ; 95\% percentile $0.17-0.18$ ) and a dog arriving during its clinical period (median 0.17 ; 95\% percentile $0.16-0.17$ ).

\section{Node 4-Probability That a Rabies-Infected Dog Disembarks in Australia}

The empirical distribution from the expert opinion workshop describing duration of stay of the fisherman had a median of 5.2 days ( $95 \%$ percentile $2.1-42.9$ days; Figure 4E). Participants noted that the duration of stay of fisherman in north-west Cape York Peninsula was influenced by success of fishing; knowledge of Australian Border Force operations; or presence of local relatives, trade contacts, and friends. The probability that an apparently healthy (incubating) dog disembarked was higher than the probability that a clinically affected dog disembarked because a dog showing overt clinical signs was believed to be more likely to be killed during travel or left on the boat on arrival and, therefore, less likely to disembark. In addition, participants noted that if the fishermen stayed $\geq 5$ days, the probability that an apparently healthy dog would disembark could be greater than if the fishermen stayed $<5$ days. Therefore, two PERT distributions were parameterized from values given in the workshop with a higher maximum value if fishermen stayed $\geq 5$ days (most likely 0.0851 , minimum 0.0213 , and maximum 0.3191 ; most likely 0.0851 , minimum 0.0213, and maximum 0.1, respectively; Figures 4F,G). The combined probability that an incubating dog disembarked had a median of 0.085 (95\% percentile $0.039-0.207$ ). The participants stated that duration of stay did not affect the probability that a clinically affected dog disembarked. Therefore, only one PERT distribution was derived from the expert opinion-regardless of duration of stay-to describe the probability of disembarkation of a clinically affected dog (most likely, minimum, and maximum estimates of 0.021, 0.021, and 0.051, respectively; Figure $4 \mathbf{H}$ ).

\section{Node 5-Probability That a Rabies-Infected} Indonesian Dog Contacts a Resident Dog (Scenario 2)

The estimated median number of daily beach-minutes that a resident dog would be on Seisia beach was 379 minutes/day (95\% percentile 171-719 minutes/day). The estimated median daily probability that a Seisia dog will be on the beach at the time an Indonesian dog disembarks was 0.26 (95\% percentile $0.12-0.50$; Figure 5).

\section{Model Outputs}

Probability of Rabies Entry into North-West Cape York Peninsula (Scenario 1) and Entry into Seisia (Scenario 2) via an Individual Indonesian Fishing Boat The estimated median probability of rabies entry into north-west Cape York Peninsula (Scenario 1) via an individual Indonesian fishing boat landing was $1.9 \times 10^{-4}$ /boat $(95 \%$ percentile $9.3 \times 10^{-6}-9.4 \times 10^{-4} /$ boat $)$ and $8.7 \times 10^{-6}(95 \%$ percentile $4.2 \times 10^{-7}-4.3 \times 10^{-5} /$ boat) for entry into Seisia (Scenario 2). These distributions are shown in Figure 6.

\section{Annual Number of Rabies-Infected Dogs and the Probability of Entry of at Least One Rabies-Infected Dog Entering per Year in North-West Cape York Peninsula (Scenario 1) and Seisia (Scenario 2)}

The estimated annual median number of dogs that arrive by boat in north-west Cape York Peninsula was 2 (95\% percentile 0-11 dogs), of which the median number of rabies-infected dogs was 0 dogs (95\% range 0-0 dogs; maximum 2 dogs). The estimated probability that at least one rabies-infected dog is introduced per year was $5.5 \times 10^{-3}\left(\mathrm{SE} 1.0 \times 10^{-3}\right)$. The estimated probability that at least one dog in the incubation period of rabies is introduced per year $\left(3.1 \times 10^{-3}\right.$; SE $\left.9.3 \times 10^{-4}\right)$ was higher than the probability that at least one dog in the clinical period of rabies is introduced per year $\left(6.4 \times 10^{-4}\right.$; SE $\left.3.4 \times 10^{-4}\right)$. The estimated median time between events of one rabies-infected dog entering north-west Cape York Peninsula is 135.3 years ( $95 \%$ percentile $4.9-715.9$ years).

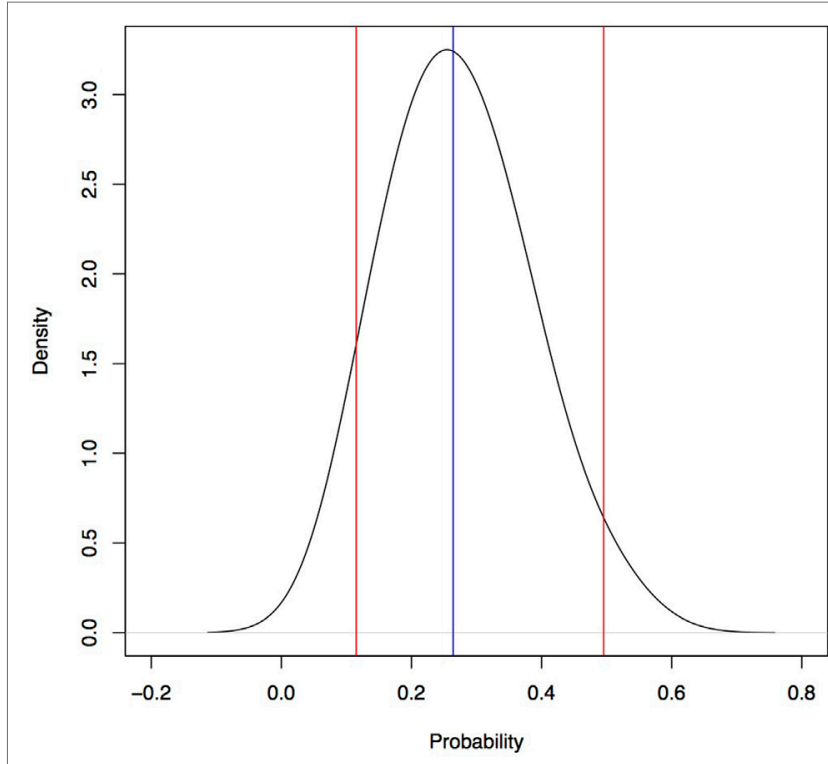

FIGURE 5 | Daily probability a dog is on the beach at Seisia used in a rabies risk assessment, north-west Cape York Peninsula, Australia. Blue line, median; red lines, 95\% percentile. 

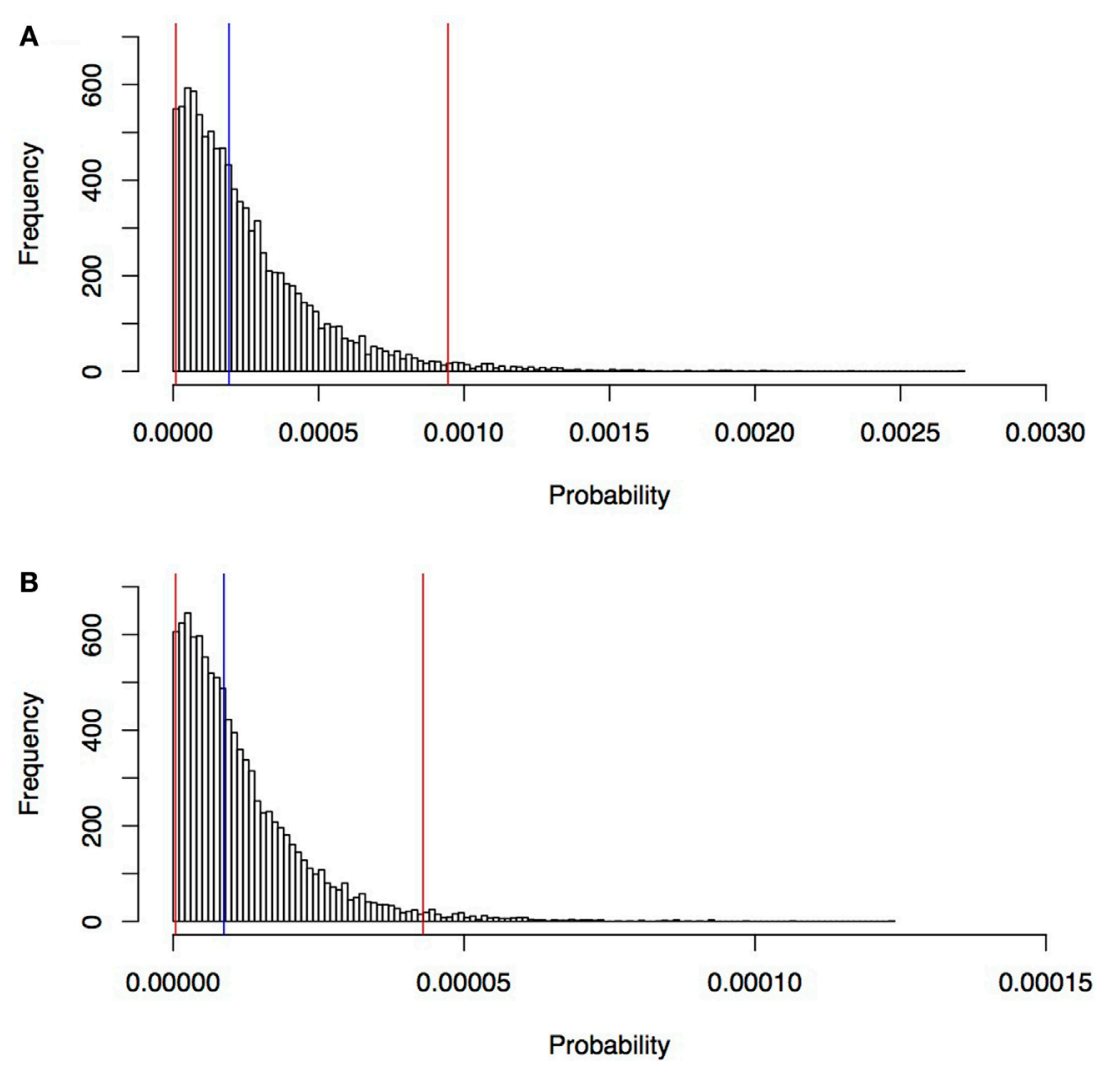

FIGURE 6 | Estimated probability of rabies entry into north-west Cape York Peninsula, Queensland (A), and into Seisia (B) via an individual Indonesian fishing boat estimated in a rabies risk assessment, north-west Cape York Peninsula, Australia. Blue line, median; red lines, 95\% percentile.

The estimated annual median number of dogs entering Seisia by boat per year was 0 ( $95 \%$ percentile $0-2$ dogs), of which the estimated median number of rabies-infected dogs that enter Seisia per year was 0 dogs (95\% range 0-0 dogs; maximum 1 dog). The estimated probability that at least one rabies-infected $\operatorname{dog}$ is introduced per year was $3.5 \times 10^{-4}\left(\mathrm{SE} 2.8 \times 10^{-4}\right)$. The estimated probability that at least one dog in the incubation period of rabies is introduced per year $\left(2.4 \times 10^{-4}\right.$; SE $\left.2.4 \times 10^{-4}\right)$ was higher than the probability that at least one dog in the clinical period of rabies is introduced per year $\left(9.9 \times 10^{-5}\right.$; SE $\left.1.4 \times 10^{-4}\right)$. The estimated median time between events of one rabies-infected dog entering Seisia is $1,724.0$ years ( $95 \%$ percentile 59.5-9,381.2 years).

\section{Introduction of Rabies to Seisia (Scenario 2)}

The estimated median probability of rabies introduction into Seisia via an individual Indonesian fishing boat landing was $4.7 \times 10^{-8} /$ boat $\left(95 \%\right.$ percentile $2.1 \times 10^{-9}-2.3 \times 10^{-7} /$ boat) and is shown in Figure 7 . The estimated probability that at least one rabies-infected dog from an Indonesian fishing boat transmits rabies to a Seisia dog per year was $8.3 \times 10^{-5}\left(\mathrm{SE} 1.4 \times 10^{-4}\right)$. The estimated median time between events of one rabies-infected dog entering and exposing rabies to a Seisia dog is $6,777.0$ years (95\% percentile 247.2-36,368.8 years).

\section{Sensitivity Analysis}

The most influential parameter on the number of infected dogs from Indonesia that enter north-west Cape York Peninsula was prevalence of rabies in Indonesia (total effect SI 0.50) followed by the probability of a dog on board a boat (total effect SI 0.48), the number of boats arriving in north-west Cape York Peninsula (total effect SI 0.46) and probability of an apparently healthy dog disembarking (total effect SI 0.39). The other inputs were less influential (total effect SI <0.25). Total effect SIs were higher than main effect SIs for all influential parameters. The total and main effect SIs are shown in Figure 8.

The most influential parameter for the probability of rabies introduction to a resident dog in Seisia was the prevalence of rabies in Indonesia (total effect SI 0.44) followed by the probability a dog is on board the boat (total effect SI 0.36), the probability of a Seisia dog on the beach (total effect SI 0.26), and the probability the infected dog developed the furious form of rabies (total effect SI 0.23). Total effect SIs were higher than main effect SIs for all influential parameters. The total and main effect SIs are shown in Figure 8.

\section{DISCUSSION}

Although the probabilities of entry of a rabies-infected dog via an illegal Indonesian fishing boat into north-west Cape York 
Peninsula, Queensland, or the subsequent transmission to a resident dog in Seisia in this region are low, the impact of a rabies incursion into this region are potentially large. Therefore, the overall risk is

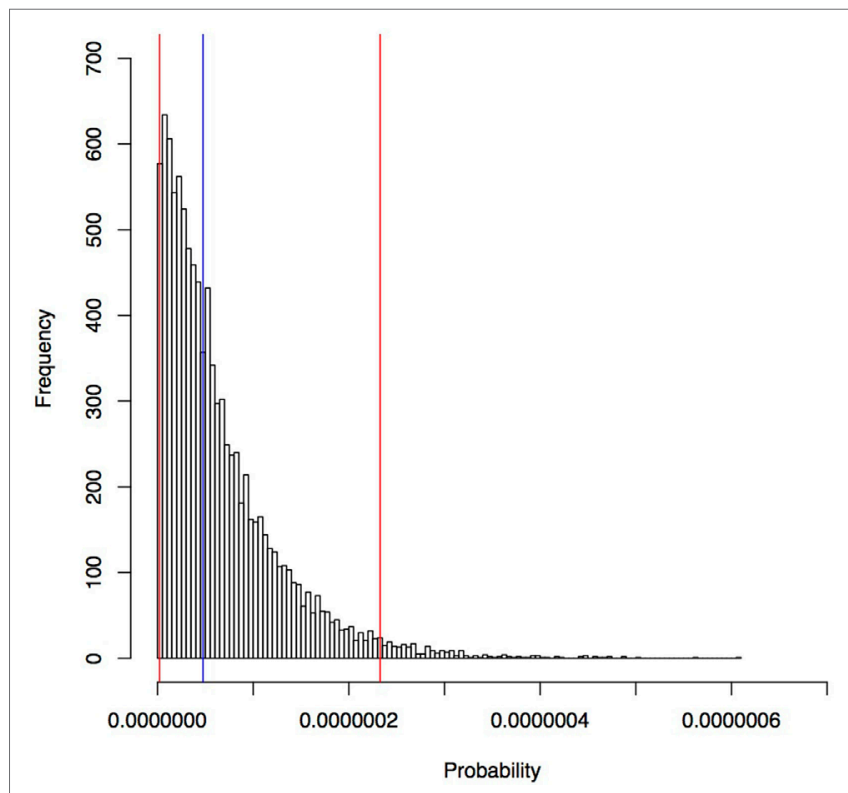

FIGURE 7 | Estimated probability of rabies introduction into Seisia via an individual Indonesian fishing boat estimated in a rabies risk assessment, north-west Cape York Peninsula, Australia. Blue line, median; red lines, 95\% percentile. not negligible. Sensitivity analysis highlighted inputs that could be targeted for further data collection to refine model outputs or surveillance and control to mitigate rabies incursion risk.

The prevalence of rabies in Indonesia was the most influential parameter on both the entry of rabies into north-west Cape York Peninsula and introduction to a resident dog in Seisia. Prevalence of rabies in the region of origin has also been found influential in other risk assessments $(14,22,23)$. The wide distribution given to this parameter reflects the variability of rabies prevalence in Indonesia and globally and the uncertainty of rabies prevalence in Indonesia due to limited surveillance. Its importance to the outcome suggests further surveillance for refinement of the model parameter and surveillance and control to help reduce rabies prevalence in Indonesia as a pre-border control for Australia. Given its high importance, vaccination campaigns to limit rabies prevalence in Indonesia would be the most effective measure to limit rabies risk of entry and transmission into northern Australian communities like the NPA compared to domestic control measures.

Some expert opinion-derived parameters had high influence on outcomes. The distributions for these parameters were wide. Although the workshop participants were targeted to achieve a sample of people with knowledge of Indonesian boat arrival into north-west Cape York Peninsula, and the structured format of the modified Delphi process was used to reduce cognitive bias (20), these wide distributions are likely to reflect uncertainty about some parameters. This also reflects the lack of documented data about these parameters. Empirical data-such as coastline surveys or surveys conducted at ports and informal ports-would
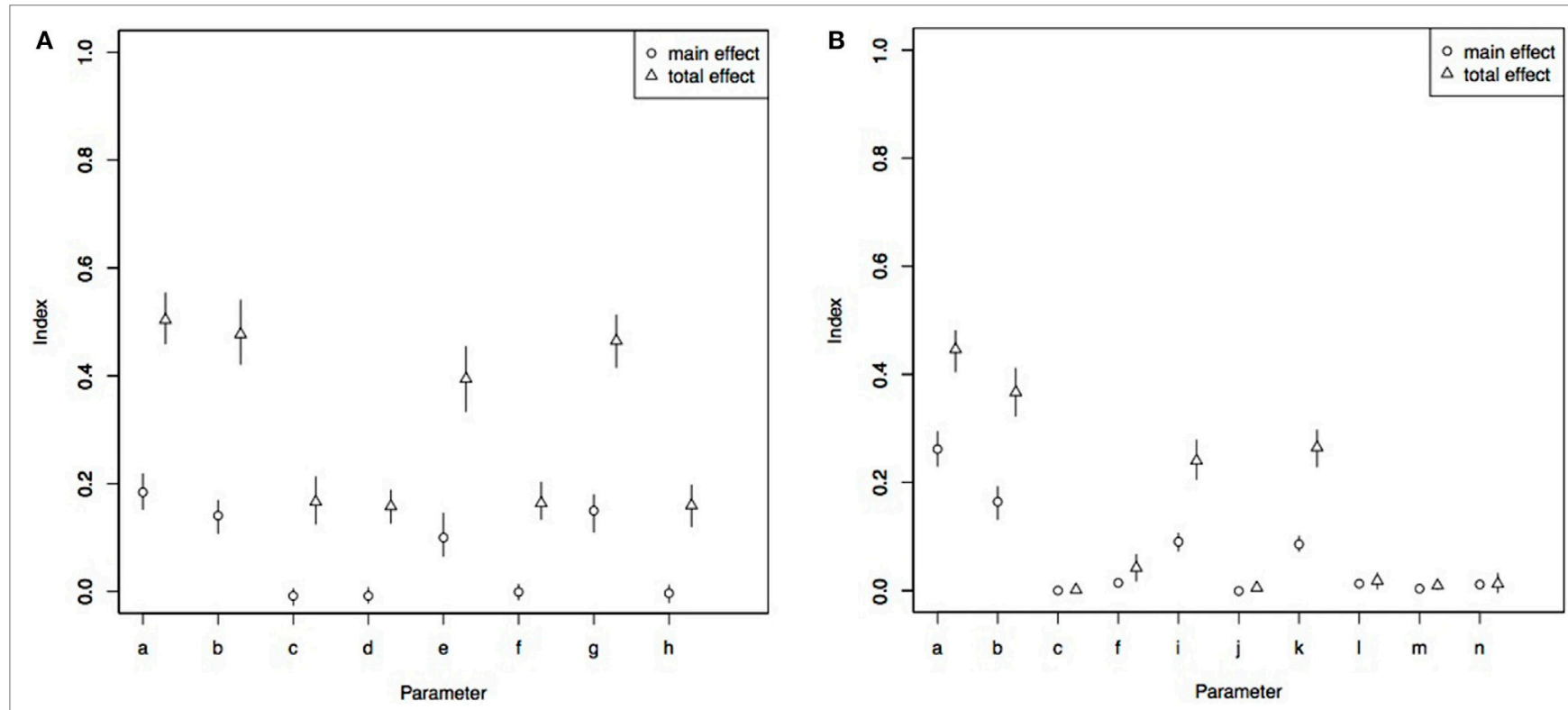

FIGURE 8 | Total and main effect sensitivity index from Sobol sensitivity analysis in a rabies risk assessment, north-west Cape York Peninsula, Australia, for number of boats likely to bring a rabies-infected dog to north-west Cape York Peninsula per year (A) and probability of rabies introduction into Seisia (B). a, prevalence of rabies in Indonesia; b, probability of at least one dog on board an Indonesian fishing boat; c, probability dogs arrives in clinical period; d, probability dog arrives in incubation period; e, probability apparently healthy dog disembarks; f, probability clinically affected dog disembarks; g, number of boats per year arriving in north-west Cape York Peninsula; h, number of dogs per boat; i, probability clinically affected dog develops furious rabies; j, probability clinically affected dog develops dumb rabies; $k$, daily probability a Seisia dog is on the beach in Seisia; I, probability a clinically affected (furious) Indonesian dog bites a Seisia dog; $\mathrm{m}$, probability a clinically affected (dumb) Indonesian dog or rabies-infected Indonesian dog in the incubation period bites a Seisia dog; n, probability Seisia dog develops rabies following bite. 
complement the expert opinion derived in this workshop. Port surveys have been conducted in Japan and in Lombok, Indonesia, and provided empirical evidence regarding the probability of a dog on board a boat, the number of dogs per boat, and the number of boats arriving from a certain origin $(22,23)$. Of the influential parameters to mitigate rabies risk, the number of boats arriving at Cape York Peninsula is perhaps the most feasible to control. Rabies entry and transmission risk through illegal Russian boat landings has been successfully reduced in Japan by limiting the number of boats entering Hokkaido via education of Russian fishermen, establishment of warning signs, daily patrols, and regular port surveillance (23). This could be beneficial for limiting the number of boats that land specifically in Seisia. However, intensive surveillance along the entirety of the coastline of north-west Cape York Peninsula would be costly. Most of the Indonesian fishing boats are landing in the sparsely populated areas of north-west Cape York Peninsula, and so the cost of surveillance would need to be balanced with the frequency of surveillance surveys required to reach an appropriate level of protection.

The inclusion of assessment of transmission to a resident Seisia dog in this study was beneficial because it highlighted further aspects of the risk pathway that could be targeted for surveillance and control for risk mitigation-the probability of meeting a Seisia dog on the beach. This differs from the study by Kwan et al. (23) who found the probability of contact with resident dogs (stray or owned) was not highly influential on the outcome; instead, the probability of contact with wildlife was influential. This difference might be due to different dog owning cultures. Most dog owners in Hokkaido keep their dogs on leads, and effective communication of rabies exposure risk has caused owners to avoid the ports. This limits exposure and subsequent transmission opportunities. In contrast, dog owners in Seisia allow their dogs to freely roam giving dogs access to the beach and therefore, a higher chance of contact with an infected dog. The probability of meeting a Seisia dog on the beach is a factor that can be controlled by limiting the resident dogs' access to Seisia beach. However, this has logistic challenges. A previous survey conducted in the NPA in 2016 showed that there was high willingness to confine domestic dogs to residents' homes by closing yard fences, chaining dogs, or keeping them inside (39). However, this was in the context of a disease outbreak and focused on short-term outbreak control strategies only. Also, confinement in yards is limited due to a lack of fencing sufficient to effectively confine dogs-many residents stated that their dogs were capable of escaping a closed yard (39). The probability of development of the furious form of rabies in clinically infected Indonesian dogs was also influential on the outcome for Scenario 2. The wide distribution given to this parameter was to reflect the high variability seen in published papers and potential uncertainty of the probability. Targeted surveillance and further data collection-including field studies of infected dogs in rabies-infected regions-could help refine this parameter and model outputs.

Pre-emptive vaccination of resident Seisia dogs against rabies could also reduce the susceptible population on the beach in Seisia and therefore, the probability of effective contact. Currently, very few rabies-free regions adopt mandatory rabies vaccination, for example, Japan and Hong Kong. Some countries-such as Malaysia-have adopted a vaccine buffer zone along shared borders with rabies-infected countries as a preventive approach (3). The scientific justification for these policies in Japan has been questioned because a change in vaccination rate and efficacy did not significantly affect the risk of a rabies introduction in a risk assessment model, even when vaccination rate was reduced to zero (23). However, there are different exposure contexts between Seisia and Hokkaido. Most dogs around the Hokkaido ports are kept on leads (23). Conversely, in Seisia, most dogs are free roaming without leads or owner supervision (39), which leads to a higher probability of contact with a foreign rabies-infected dog. Also, the population with the greatest influence on risk of exposure in Hokkaido was wildlife not domestic dogs, which could also affect the influence of domestic dog vaccination in their study (23). Further research into the effects of a pre-emptive vaccination program on the reduction of rabies entry and exposure risk in Seisia could be used to investigate the benefits of a pre-emptive vaccination program in the NPA.

This study did not consider the exposure risk of wild-living dogs (domestic dogs and dingoes), which could lead to underestimation of the probability of rabies transmission in this study. Wild-living dogs inhabit most of Australia (40), including the sparsely populated north-west Cape York Peninsula and are known to frequent NPA communities (41). However, the lack of data on their abundance and distribution limits inclusion of wildliving dog transmission in a risk assessment for this region. Also, the current study focused on domestic dogs in Seisia due to the risk that this poses to public health. Inclusion of the probability of transmission to wild-living dogs in a future risk assessment of rabies in north-west Cape York Peninsula (including Seisia) would be beneficial for a more robust rabies risk assessment in which-similar to the risk assessment for rabies introduction into Hokkaido, Japan-controlling exposure and transmission into the these wild populations could be more influential than controlling exposure and transmission in the domestic dogs in north-west Cape York Peninsula.

There are limitations associated with the current study. We did not differentiate variance of model input and outputs into uncertainty and natural variability, due to insufficient empirical data about most of the parameters. A two-dimensional risk assessment in which uncertainty and variability are differentiated can aid interpretation of the sensitivity analysis. For example, if the variance of influential parameters is mainly due to uncertainty, more research to identify natural variability and its influence on risk is likely to be beneficial before recommendations for control measures are made. However, if the variance of influential parameters is mainly due to natural variability, recommendations for control measures that change the circumstances (for example, reducing number of dogs available for exposure and transmission) are likely to reduce risk. Other limitations arise from the analysis of GPS coordinate field data to estimate the daily probability a Seisia dog would be on the beach at the time of arrival of the Indonesian dog. This analysis did not consider that more than one dog could be present on the beach at any one 
point in time because each dog was considered separately. This could either underestimate the probability of contact (a greater number of dogs on the beach is likely to increase the opportunity for contact) or overestimate the probability of contact (overlapping minutes were counted twice, and consequently, the number of beach-minutes/day was higher). Also, the dogs that were GPS tracked were only tracked for short periods (median 3 days; range 1-13 days). This could underestimate the number of dogs that visit the beach, and subsequently probability of contact, because dogs that usually visit the beach may not have visited during GPS tracking. This was an influential parameter on the probability of rabies entry and exposure into Seisia; therefore, further surveys on dog roaming patterns along the beach could refine model outputs to produce a more accurate assessment of the risk of transmission of rabies to a resident Seisia dog. We also made the assumption that an Indonesian fishing boat would leave Seisia beach and take the dog with it. This could underestimate the probability of entry and introduction because for longer durations of stay or if the dog remained indefinitely in Seisia, the probability that a dog arriving during the incubation phase becomes clinical also increases, therefore increasing the number of dogs available to infect resident Seisia dogs. Also, the possibility that dogs could die during the incubation period of other causes-thus reducing the number of dogs arriving and disembarking-was not accounted for in the current model, which could consequently overestimate the risk of rabies entry. The assumption only clinically infected dogs will transmit rabies could also slightly underestimate the risk of rabies transmission into Seisia. In some cases, there is a short preclinical infectious period in which a dog is infectious but does not show clinical signs, which would increase the number of dogs capable of transmitting rabies and therefore the probability of transmission. This is unlikely to affect the overall results because it is generally very short leading to only a few more dogs being infectious, and the model was not sensitive to the probability of a dog arriving in the incubation or clinical stage of infection.

\section{REFERENCES}

1. Hampson K, Coudeville L, Lembo T, Sambo M, Kieffer A, Attlan M, et al. Estimating the global burden of endemic canine rabies. PLoS Negl Trop Dis (2015) 9(4):e0003709. doi:10.1371/journal.pntd.0003709

2. WHO. World Health Organization expert consultation on rabies: Second report. World Health Organ Tech Rep Ser (2013) 982:1-139, back cover.

3. Tenzin, Ward MP. Review of rabies epidemiology and control in South, South East and East Asia: past, present and prospects for elimination. Zoonoses Public Health (2012) 59(7):451-67. doi:10.1111/j.1863-2378.2012.01489.x

4. Putra AA, Hampson K, Girardi J, Hiby E, Knobel D, Mardiana IW, et al. Response to a rabies epidemic, Bali, Indonesia, 2008-2011. Emerg Infect Dis (2013) 19(4):648-51. doi:10.3201/eid1904.120380

5. Tenzin, Sharma B, Dhand NK, Timsina N, Ward MP. Reemergence of rabies in Chukka District, Bhutan, 2008. Emerg Infect Dis (2010) 16(12):1925-30. doi:10.3201/eid1612.100958

6. Tenzin, Dhand NK, Dorjee J, Ward MP. Re-emergence of rabies in dogs and other domestic animals in eastern Bhutan, 2005-2007. Epidemiol Infect (2011) 139(2):220-5. doi:10.1017/S0950268810001135

7. Yin CP, Zhou H, Wu H, Tao XY, Rayner S, Wang SM, et al. Analysis on factors related to rabies epidemic in China from 2007-2011. Virol Sin (2012) 27(2):132-43. doi:10.1007/s12250-012-3244-y

\section{CONCLUSION}

This study revealed a low but not negligible risk of rabies entry into north-west Cape York Peninsula, Queensland, and introduction to a resident dog in Seisia via the transport of an infected dog on an Indonesian fishing boat. The most influential input in both sensitivity analyses was the prevalence of rabies entry and exposure in Seisia was the prevalence of rabies in Indonesia, which is a reflection of the uncertainty and variability of this parameter. Further data on the prevalence as well as other influential parameters such as the number of boats arriving and the probability of a dog on board would improve accuracy of model outputs. Furthermore, limiting the risk of rabies exposure could be achieved by limiting Seisia dogs' access to the beach or reducing the susceptible population by vaccination.

\section{ETHICS STATEMENT}

The protocol was approved and subsequently carried out in accordance to recommendations given by the Human Research Ethics Committee of The University of Sydney (reference number 2016/711) with written informed consent from all participants.

\section{AUTHOR CONTRIBUTIONS}

The study design, data collection, and manuscript writing and revision were completed by all authors (EH, VB, and MW). Data analysis and interpretation were completed by $\mathrm{EH}$ with contributions from VB and MW.

\section{ACKNOWLEDGMENTS}

The authors thank the participants from the Northern Peninsula Area, Queensland for their involvement and contribution to this study.

8. Yao H-W, Yang Y, Liu K, Li X-L, Zuo S-Q, Sun R-X, et al. The spatiotemporal expansion of human rabies and its probable explanation in mainland China, 2004-2013. PLoS Negl Trop Dis (2015) 9(2):e0003502. doi:10.1371/journal. pntd.0003502

9. Townsend SE, Lembo T, Cleaveland S, Meslin FX, Miranda ME, Putra AAG, et al. Surveillance guidelines for disease elimination: a case study of canine rabies. Comp Immunol Microbiol Infect Dis (2013) 36(3):249-61. doi:10.1016/j. cimid.2012.10.008

10. Ward MP, Hernández-Jover M. A generic rabies risk assessment tool to support surveillance. Prev Vet Med (2015) 120(1):4-11. doi:10.1016/j. prevetmed.2014.11.005

11. Goddard AD, Donaldson NM, Horton DL, Kosmider R, Kelly LA, Sayers AR, et al. A quantitative release assessment for the noncommercial movement of companion nnimals: risk of rabies reintroduction to the United Kingdom. Risk Anal (2012) 32(10):1769-83. doi:10.1111/j.1539-6924.2012.01804.x

12. Jones RD, Kelly L, Fooks AR, Wooldridge M. Quantitative Risk assessment of rabies entering Great Britain from North America via cats and dogs. Risk Anal (2005) 25(3):533-42. doi:10.1111/j.1539-6924.2005.00613.x

13. Ramnial V, Kosmider R, Aylan O, Freuling C, Müller T, Fooks AR. Quantitative risk assessment to compare the risk of rabies entering the UK from Turkey via quarantine, the Pet Travel Scheme and the EU Pet Movement Policy. Epidemiol Infect (2010) 138(8):1114-25. doi:10.1017/S0950268809991221 
14. Weng H-Y, Wu P-I, Yang P-C, Tsai Y-L, Chang C-C. A quantitative risk assessment model to evaluate effective border control measures for rabies prevention. Vet Res (2010) 41(1):11. doi:10.1051/vetres/2009059

15. Windiyaningsih C, Wilde H, Meslin FX, Suroso T, Widarso HS. The rabies epidemic on Flores Island, Indonesia (1998-2003). J Med Assoc Thai (2004) 87(11):1389-93.

16. Mahardika GNK, Dibia N, Budayanti NS, Susilawathi NM, Subrata K, Darwinata $\mathrm{AE}$, et al. Phylogenetic analysis and victim contact tracing of rabies virus from humans and dogs in Bali, Indonesia. Epidemiol Infect (2014) 142(6):1146-54. doi:10.1017/S0950268813002021

17. Sparkes J, Fleming PJ, Ballard G, Scott-Orr H, Dürr S, Ward MP. Canine rabies in Australia: a review of preparedness and research needs. Zoonoses Public Health (2014) 63:237-53. doi:10.1111/zph.12142

18. Brookes VJ, Ward MP. Expert opinion to identify high-risk entry routes of canine rabies into Papua New Guinea. Zoonoses Public Health (2016) 64(2):156-60. doi:10.1111/zph.12284

19. Bedford T, Cooke R. Probabilistic Risk Analysis: Foundations and Methods. Cambridge: Cambridge University Press (2001).

20. Speirs-Bridge A, Fidler F, McBride M, Flander L, Cumming G, Burgman M. Reducing overconfidence in the interval judgments of experts. Risk Anal (2010) 30(3):512-23. doi:10.1111/j.1539-6924.2009.01337.x

21. Dürr S, Ward MP. Roaming behaviour and home range estimation of domestic dogs in Aboriginal and Torres Strait Islander communities in northern Australia using four different methods. Prev Vet Med (2014) 117(2):340-57. doi:10.1016/j.prevetmed.2014.07.008

22. Mustiana A. Assessment of the Risk for Rabies Introduction and Establishment in Lombok, Indonesia [Masters Thesis]. Masters of Veterinary Science, The University of Sydney (2013).

23. Kwan NCL, Ogawa H, Yamada A, Sugiura K. Quantitative risk assessment of the introduction of rabies into Japan through the illegal landing of dogs from Russian fishing boats in the ports of Hokkaido, Japan. Prev Vet Med (2016) 128:112-23. doi:10.1016/j.prevetmed.2016.04.015

24. Kitala PM, McDermott JJ, Kyule MN, Gathuma JM. Community-based active surveillance for rabies in Machakos district, Kenya. Prev Vet Med (2000) 44(1):73-85. doi:10.1016/S0167-5877(99)00114-2

25. Cleaveland S, Kaare M, Tiringa P, Mlengeya T, Barrat J. A dog rabies vaccination campaign in rural Africa: impact on the incidence of dog rabies and human dog-bite injuries. Vaccine (2003) 21(17-18):1965-73. doi:10.1016/ S0264-410X(02)00778-8

26. Kayali U, Mindekem R, Yémadji N, Oussiguéré A, Naïssengar S, Ndoutamia AG, et al. Incidence of canine rabies in N'Djaména, Chad. Prev Vet Med (2003) 61(3):227-33. doi:10.1016/j.prevetmed.2003.07.002

27. Tojinbara K, Sugiura K, Yamada A, Kakitani I, Kwan NCL, Sugiura K. Estimating the probability distribution of the incubation period for rabies using data from the 1948-1954 rabies epidemic in Tokyo. Prev Vet Med (2016) 123:102-5. doi:10.1016/j.prevetmed.2015.11.018

28. Hampson K, Dushoff J, Cleaveland S, Haydon DT, Kaare M, Packer C, et al. Transmission dynamics and prospects for the elimination of canine rabies. PLoS Biol (2009) 7(3):e53. doi:10.1371/journal.pbio.1000053
29. Vaughn JB Jr, Gerhardt P, Newell KW. Excretion of street rabies virus in the saliva of dogs. JAMA (1965) 193(5):363-8. doi:10.1001/jama.1965. 03090050039010

30. Fekadu M, Shaddock JH. Peripheral distribution of virus in dogs inoculated with two strains of rabies virus. Am J Vet Res (1984) 45(4):724-9.

31. Foggin CM. Rabies and Rabies-Related Viruses in Zimbabwe: Historical, Virological and Ecological Aspects. Doctor of Philosophy (Ph. D.), University of Zimbabwe (1988).

32. McBride MF, Garnett ST, Szabo JK, Burbidge AH, Butchart SHM, Christidis L, et al. Structured elicitation of expert judgments for threatened species assessment: a case study on a continental scale using email. Methods Ecol Evol (2012) 3(5):906-20. doi:10.1111/j.2041-210X.2012.00221.x

33. Catley A, Alders RG, Wood JLN. Participatory epidemiology: approaches, methods, experiences. Vet J (2012) 191(2):151-60. doi:10.1016/j.tvjl.2011. 03.010

34. Pujol G, Iooss B, Janon A, Boumhaout K, Da Veiga S, Fruth J, et al. Sensitivity: Global Sensitivity Analysis of Model Outputs. R Package Version 1.13.0. ed. (2016).

35. Davison AC, Hinkley DV. Bootstrap Methods and Their Application. Cambridge: Cambridge University Press (1997).

36. Canty A, Ripley B. Boot: Bootstrap R (S-Plus) Functions, R Package Version 1.3-18. (2016).

37. Dürr S, Dhand NK, Bombara C, Molloy S, Ward MP. What influences the home range size of free-roaming domestic dogs? Epidemiol Infect (2017) 145:1-12. doi:10.1017/S095026881700022X

38. Fekadu M. Pathogenesis of rabies virus infection in dogs. Rev Infect Dis (1988) 10(4):S678-83. doi:10.1093/clinids/10.Supplement_4.S678

39. Hudson EG, Dhand NK, Dürr S, Ward MP. A survey of dog owners in remote northern Australian indigenous communities to inform rabies incursion planning. PLoS Negl Trop Dis (2016) 10:e0004649. doi:10.1371/journal.pntd. 0004649

40. Allen BL, West P. Influence of dingoes on sheep distribution in Australia. Aust Vet J (2013) 91(7):261-7. doi:10.1111/avj.12075

41. Gabriele-Rivet V, Arsenault J, Brookes V, Ward M. Studies on wild dogs and dingoes to inform disease spread modelling. Presented at Australian and New Zealand College of Veterinary Scientists: Science Week Conference 6th-8th July 2017. (2017).

Conflict of Interest Statement: The authors declare that the research was conducted in the absence of any commercial or financial relationships that could be construed as a potential conflict of interest.

Copyright (c) 2017 Hudson, Brookes and Ward. This is an open-access article distributed under the terms of the Creative Commons Attribution License (CC BY). The use, distribution or reproduction in other forums is permitted, provided the original author(s) or licensor are credited and that the original publication in this journal is cited, in accordance with accepted academic practice. No use, distribution or reproduction is permitted which does not comply with these terms. 